
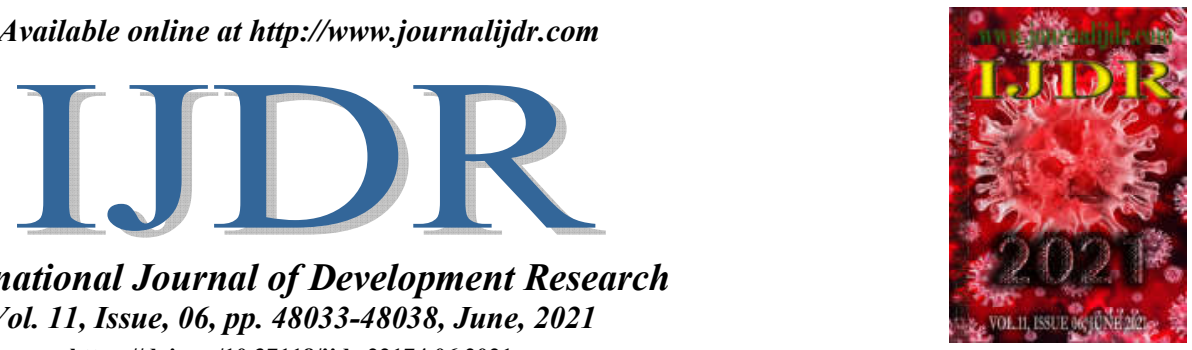

ISSN: 2230-9926

International Journal of Development Research

Vol. 11, Issue, 06, pp. 48033-48038, June, 2021

https://doi.org/10.37118/ijdr.22174.06.2021

\title{
ANÁLISE ESPACIAL E EPIDEMIOLÓGICA DOS CASOS NOTIFICADOS DE LEISHMANIOSE VISCERAL HUMANA EM ÁREA ENDÊMICA, NO ESTADO DO MARANHÃO, BRASIL
}

\author{
Josemeire da Costa Ximenes ${ }^{1,}{ }^{*}$, Joseneide Teixeira Câmara ${ }^{2}$, Ana Paula Casseb ${ }^{3}$, Beatriz Mourão \\ Pereira $^{4}$, Aline Raquel de Sousa Ibiapina ${ }^{5}$, Haylane Nunes da Conceição ${ }^{6}$, Marília Ramalho \\ Oliveira ${ }^{7}$, Rayssa Stefani Cesar Lima ${ }^{8}$, Magnólia de Jesus Sousa Magalhães ${ }^{9}$, Ana Carla Marques \\ da Costa ${ }^{10}$, Amanda Laurindo Monteiro ${ }^{11}$ and Felipe Santana e Silva ${ }^{12}$
}

${ }^{1}$ Farmacêutica, mestranda em Biodiversidade, Ambiente e Saúde pela Universidade Estadual do Maranhão; ${ }^{2}$ Doutora em medicina tropical, professora adjunta da Universidade Estadual do Maranhão; ${ }^{3}$ Enfermeira pela

Universidade Estadual do Maranhão; ${ }^{4}$ Enfermeira, mestre em Biodiversidade, Ambiente e Saúde pela Universidade Estadual do Maranhão e professora substituta da Universidade Estadual do Maranhão; ${ }^{5}$ Doutora em Enfermagem e Professora do curso de enfermagem da Universidade Federal do Piauí; ${ }^{6}$ Acadêmica do curso Bacharelado em Fisioterapia do Centro Universitário de Ciências e Tecnologia do Maranhão; ${ }^{7}$ Enfermeira pela

Universidade Estadual do Maranhão; ${ }^{8}$ Acadêmica do curso de enfermagem pela Universidade Estadual do Maranhão; 9 Doutora em Biologia Celular e Molecular Aplicada à Saúde, docente da Universidade Estadual do Maranhão; ${ }^{10}$ Doutora em Biologia Celular e Molecular Aplicada à Saúde, docente da Universidade Estadual do Maranhão; ${ }^{11}$ Doutor em Ciências Biológicas (Microbiologia), docente da Universidade Estadual do Maranhão;

${ }^{12}$ Enfermeiro, mestrando em Biodiversidade, Ambiente e Saúde da Universidade Estadual do Maranhão

\section{ARTICLE INFO}

Article History:

Received $03^{\text {rd }}$ March, 2021

Received in revised form

$10^{\text {th }}$ April, 2021

Accepted $21^{\text {st }}$ May, 2021

Published online $30^{\text {th }}$ June, 2021

Key Words:

Leishmaniose Visceral,

Epidemiologia, Análise espaço- Temporal.

*Corresponding author:

Josemeire da Costa Ximenes

\begin{abstract}
Introdução: A Leishmaniose Visceral (LV) é uma doença endêmica no Brasil, no entanto têm sido registrados surtos frequentes, sendo estimada que cause a nona maior carga de doenças entre as patologias infecciosas individuais. Objetivo: analisar os dados clínicos- epidemiológicos e a distribuição espaço-temporal dos casos notificados de LV humana, no município de CaxiasMaranhão. Métodos: trata-se de um estudo de caráterdescritivo-exploratório com abordagem quantitativa, de recorte transversal no período de 2014 a 2019, realizada em Caxias, Maranhão localizada na Região Nordeste do Brasil. Foi utilizada técnica de geoprocessamento através do software QGIS 2.18, para a análise espacial dos casos confirmados de LV. Resultados: Foram investigados 535 casos, com 33,1\% $(n=177)$ confirmados. Verificou-se maior frequência da doença no sexo masculino com baixo nível de escolaridade, sendo a faixa etária de 1 a 9 anos considerada como fator de risco para LV. A análise espaço-temporal reveloua distribuição geográfica da LV em todo o território municipal. Conclusão: o perfil clínico-epidemiológico dos casos de LVH ocorridos no munícipio, assemelham-se ao descrito na literatura nacional, as ferramentas de geoprocessamentomostram-se úteis ao permitir o mapeamento das áreas, possibilitando identificar áreas de elevada prioridade de intervenção e planejamento em saúde.
\end{abstract}

Copyright (C) 2021, Josemeire da Costa Ximenes et al. This is an open access article distributed under the Creative Commons Attribution License, which permits unrestricted use, distribution, and reproduction in any medium, provided the original work is properly cited.

Citation: Josemeire da Costa Ximenes, Joseneide Teixeira Câmara, Ana Paula Casseb, Beatriz Mourão Pereira et al. "Análise espacial e epidemiológica dos casos notificados de leishmaniose visceral humana em área endêmica, no estado do maranhão, Brasil”, International Journal of Development Research, 11, (06), 48033-48038. 


\section{INTRODUCTION}

Considerada um problema de saúde pública, a Leishmaniose Visceral (LV) é uma doença endêmica no Brasil, causada por protozoários do gênero Leishmania, de transmissão vetorial, principalmente pela fêmea infectada do mosquitoLutzomyialongipalpis, podendo ser letal em $90 \%$ dos casos não tratados. Éconsiderada uma doença infecciosa, sistêmica, caracterizada por episódios de febre de longa duração, anemia, perda substancial de peso, astenia, aumento do baço e do fígado(Brasil, 2019, Who, 2020,Vianaet al.,2017). No mundo, estimase que ocorra anualmente, 300.000 milhões de casos de LV com cerca de 20.000 óbitos. Com aproximadamente 1 bilhão de pessoas vivendo em áreas endêmicas com risco de infecção (616 milhões por LV e 431 milhões por Leishmaniose Tegumentar(LT)), e registrando em 2017, 20.792 dos 22.145 (94\%) de novos casos ocorridos em sete países: Brasil, Etiópia, Índia, Quênia, Somália, Sudão do Sul e Sudão. Mesmo com grande parte dos casos de LV subnotificados, o Brasil é responsável por mais de $90 \%$ dos casos notificados de Leishmaniose Visceral Humana(LVH) $>95 \%$ na América Latina (Who, 2020; Lana et al., 2018). Quanto aos aspectos epidemiológicos, a doença passou do caráter predominantemente rural, para expansão em áreas urbanas de médio e grande porte, reconhecida como urbanização da LV, favorecida pelas mudanças socioambientais, imigração da população humana e canina portadores do parasito para áreas não endêmicas, fatores climáticos e ação antrópica do homem (da Conceição Araújo, 2017,Brasil, 2014, ZubeneDonalísio,2016). É uma doença de notificação compulsória, com características de evolução grave, apresentando um grande número de óbitos, apesar de possuir tratamento disponível no Sistema Único de Saúde (SUS). O diagnóstico precisa ser realizado o mais precocemente possível sendo necessário ser oferecido de forma obrigatória em todas as áreas com transmissão ou com risco de transmissão (Brasil,2019, Alves eFonseca, 2018).

Embora seja estimada como causador da nona maior carga de doenças entre as doenças individuais, a Leishmaniose continua ausente nos debates sobre as prioridades das doenças tropicais, por estar diretamente associada à pobreza, à desnutrição, deslocamento da população, moradia precária, sistema imunológico imunossuprimidos e a escassez de recursos, principalmente afetando pessoas pobres na África, Ásia e América Latina (Alvar et al., 2012, Who, 2020,de Souza et al., 2020,Marcondes e Rossi, 2013). O Programa de Vigilância e Controle da Leishmaniose Visceral (PVCLV) baseia-se em medidas de diagnóstico e tratamento precoce dos casos humanos, redução da população de flebotomíneos, eliminação de reservatórios associadas a ações de educação em saúde (Brasil, 2014, Opas, 2019). A investigação dos casos, estratificação de riscoe as análises epidemiológicas devem ser utilizadas como estratégias para o desenvolvimento de ações eficientes e oportunas.

Dessa maneira, conhecer as áreas de maior incidência ou de maior risco de casos é fundamental para a adequação de recursos e direcionamento das ações. Sendo as análises e a distribuição espaçotemporal dos casos utilizadas para o entendimento da dinâmica da transmissão da LV, tendo o georreferenciamento como ferramenta essencial possibilitandovisualizar os cenários de distribuição da doença(Furtadoet al., 2015,da Conceição Araújo, 2017,Souza et al., 2020). Diante desse contexto de vulnerabilidade, o Brasil ainda se encontrano cenário de transição epidemiológica e demográfica marcado pela predominância concomitante de doenças transmissíveis e crônico-degenerativas, além da subnotificação de dados, que dificulta o planejamento das ações para o controle da LV, uma vez que é necessário melhor definir as áreas de transmissão ou de risco. Por fazer parte do rol das doenças consideradas negligenciadas, constitui-se ainda em um dos grandes desafios no âmbito da saúde, sendo que as medidas de controle usualmenteexecutadas precisam ser integradas para que possam ser efetivas. Desta forma, essa pesquisa tem como objetivo, analisar os aspectosclínicos-epidemiológicos e a distribuição espacial dos casos notificados de LVH, no município de Caxias-Maranhão.

\section{METODOLOGIA}

Trata-se de um estudo descritivo-exploratório de abordagem quantitativa, com recorte no período de 2014 a 2019. A população do estudo foi composta por 535casos notificados de LVH em residentes de Caxias, Maranhão, localizada na Região Nordeste do Brasil. Esse levantamento foi feito por meio das análisesdas fichas de notificações dos casos de LV na Vigilância Epidemiológica do município. O instrumento de coleta de dados foi uma ficha adaptada baseada nas informações presentes nas fichas de notificação de LV do Ministério da Saúde das seguintes variáveis: sexo, faixa etária, raça, escolaridade, zona de moradia, ocupação, manifestações clínicas, droga inicial utilizada, coinfecção HIV, diagnóstico imunológico, diagnóstico parasitológico, tipo de entrada, critério de confirmação, doença relacionada ao trabalho e evolução do caso. Os dados foram tabulados e analisados no software estatístico Epi Info, versão 7.2.1.0. Para caracterização da amostra foi usado distribuições de frequência absoluta e relativa e medidas de tendência central e dispersão. $\mathrm{Na}$ análise bivariada, utilizou-se o teste Qui-quadrado de Pearson e Exato de Fisher, com intervalo de confiança de $95 \%\left(\mathrm{IC}_{95 \%}\right)$. Para a análise espacial dos casos de LV foram construídos mapas, os quais representam a ocorrência de casos confirmados da doença no período do estudo. A elaboração dos mapas foi realizada através de um software de geoprocessamento denominado QGIS 2.18. Os dados foram coletados e plotados em suas respectivas coordenadas geográficas em sistema de projeção SIRGAS 200, o projeto foi salvo em formato Jpeg em resolução de 500 dpi. A base cartográfica utilizada foi do IBGE 2016. O estudo foi desenvolvido após aprovação pelo comitê de ética em pesquisa com seres humanos da Universidade Estadual do Maranhão (UEMA) sob o parecer de número 3.235.493.

\section{RESULTADOS}

Foram investigados535 casos de LV. Destes, 33,1\% $(\mathrm{n}=177)$ foram confirmados, $352(65,8 \%)$ descartados e $6(1,1 \%)$ casos com a classificação final ignorada.No período do estudo, observou-se maior prevalência nos anos de 2014 e 2017, com 27,1\% e 21,5\% respectivamente, seguido de 2015 com 18,6\%, o ano de 2016 apresentando 16,9\%, 2018 com 12,4\% e por fim um declínio em 2019 com apenas 3,4podendo, porém está associado a subnotificação dos casos. Quanto ao perfil sociodemográfico, através da regressão logística binária, identificou-se que estar na faixa etária de 1 a 9 anos e ter como profissão ser agropecuário, é fator de risco para LV. As demais variáveis não obtiveram significância estatística, mas observou-se que 58,2\% ( $\mathrm{n}=103)$ dos casos são do sexo masculino, $57,7 \%(\mathrm{n}=41)$ com até 8 anos de estudo, $73,8 \%(\mathrm{n}=127)$ residem na zona urbana e $82,4 \%(n=145)$ se autodeclararam pardos (Tabela 1). Os sintomas mais frequentes nos casos confirmados de LV foram: febre, fraqueza, palidez, emagrecimento e esplenomegalia. Na tabela 2 , observou-seque $83,1 \% \quad(n=147)$ dos casos notificados de LVutilizaram o antimonial pentavalente, com $9 \%(n=16)$ convivendo na coinfecção com HIV. No que concerne ao diagnóstico em 94,9\% $(n=168)$ dos casos receberam confirmação laboratorial, sendo que $35 \%(\mathrm{n}=62)$ foram diagnosticados através do exame imunológico e $23,2 \%(n=41)$ do parasitológico, com 94,4\% $(n=167)$ das notificações como casos novos, destes $59,3 \%(\mathrm{n}=105)$ evoluíram para cura e $10,2 \%$ $(\mathrm{n}=18)$ faleceram por LV.

\section{DISCUSSÃO}

Verificou-se que os anos de 2014 e 2017 foram os que registraram maior número de casos de LVH. O Maranhão encontra-se entre os estados da federação com maior número de casos da doença. Entre os anos de 1999 a 2005, o estado liderou em número de casos confirmados no Brasil, com registroaté 2009, de 9.972 notificações, sendo a maioriaproveniente dos municípiosquecompõem a grandeIlha de São Luís(Furtado et al., 2015, BRASIL, 2019). A redução dos casos de LVH no período do estudo, pode estar associado a medidas simples como a limpeza de quintais e terrenos, contribuindo para 
Tabela 1. Análise univariada de regressão da classificação final com o perfil sociodemográfico dos casos notificados com Leishmaniose Visceral Humana em Caxias-MA, 2014/2019

\begin{tabular}{|c|c|c|c|c|c|c|}
\hline \multicolumn{7}{|c|}{ Classificação final } \\
\hline \multirow[t]{2}{*}{ Variáveis } & \multicolumn{2}{|c|}{ Descartado } & \multicolumn{2}{|c|}{ Confirmado } & \multirow[t]{2}{*}{$x^{2}$} & \multirow[t]{2}{*}{$\mathrm{p}$} \\
\hline & $\mathrm{n}$ & $\%$ & $\mathrm{n}$ & $\%$ & & \\
\hline \multicolumn{7}{|l|}{ Faixa Etária } \\
\hline Menos de 1 ano & 44 & $(12,5)$ & 18 & $(10,2)$ & 17,811 & $0,003^{\mathrm{a}}$ \\
\hline De 1 a 9 anos & 161 & $(45,7)$ & 70 & $(39,5)$ & & \\
\hline De 10 a 19 anos & 29 & $(8,2)$ & 8 & $(4,5)$ & & \\
\hline De 20 a 39 anos & 38 & $(10,8)$ & 34 & $(19,2)$ & & \\
\hline De 40 a 59 anos & 39 & $(11,1)$ & 34 & $(19,2)$ & & \\
\hline 60 anos ou mais & 41 & $(11,6)$ & 13 & $(7,3)$ & & \\
\hline \multicolumn{7}{|l|}{ Gênero } \\
\hline Feminino & 138 & $(39,2)$ & 74 & $(41,8)$ & 0,332 & $0,564^{\mathrm{a}}$ \\
\hline Masculino & 214 & $(60,8)$ & 103 & $(58,2)$ & & \\
\hline \multicolumn{7}{|l|}{ Escolaridade } \\
\hline Analfabeto & 7 & $(5,9)$ & 5 & $(7,0)$ & 2,361 & $0,307^{\mathrm{a}}$ \\
\hline Até 8 anos de estudo & 81 & $(68,6)$ & 41 & $(57,7)$ & & \\
\hline Mais de 8 anos de estudo & 30 & $(25,4)$ & 25 & $(35,2)$ & & \\
\hline \multicolumn{7}{|l|}{ Zona } \\
\hline Urbana & 266 & $(76,0)$ & 127 & $(73,8)$ & 0,290 & $0,590^{\mathrm{a}}$ \\
\hline Rural & 84 & $(24,0)$ & 45 & $(26,2)$ & & \\
\hline \multicolumn{7}{|l|}{ Raça } \\
\hline Branca & 46 & $(13,1)$ & 13 & $(7,4)$ & 5,663 & $0,129^{\mathrm{a}}$ \\
\hline Preta & 33 & $(9,4)$ & 17 & $(9,7)$ & & \\
\hline Amarela & 7 & $(2,0)$ & 1 & $(, 6)$ & & \\
\hline Parda & 266 & $(75,6)$ & 145 & $(82,4)$ & & \\
\hline \multicolumn{7}{|l|}{ Gestante } \\
\hline Sim & 1 & $(2,5)$ & - & - & - & $1,000^{\mathrm{b}}$ \\
\hline Não & 39 & $(97,5)$ & 17 & $(100,0)$ & & \\
\hline \multicolumn{7}{|l|}{ Ocupação } \\
\hline Estudante & 46 & $(39,7)$ & 13 & $(18,8)$ & 16,451 & $0,002^{\mathrm{a}}$ \\
\hline Agropecuário & 19 & $(16,4)$ & 23 & $(33,3)$ & & \\
\hline Aposentado & 21 & $(18,1)$ & 6 & $(8,7)$ & & \\
\hline Dona de casa & 10 & $(8,6)$ & 9 & $(13,0)$ & & \\
\hline Outros & 20 & $(17,2)$ & 18 & $(26,1)$ & & \\
\hline
\end{tabular}

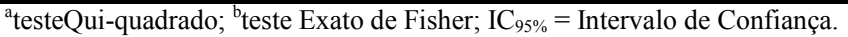

Nota: ignorados e não respondentes foram excluídos da análise de regressão.

Fonte: Secretaria de Saúde de Caxias-Ma, Departamento de Vigilância Epidemiológica.

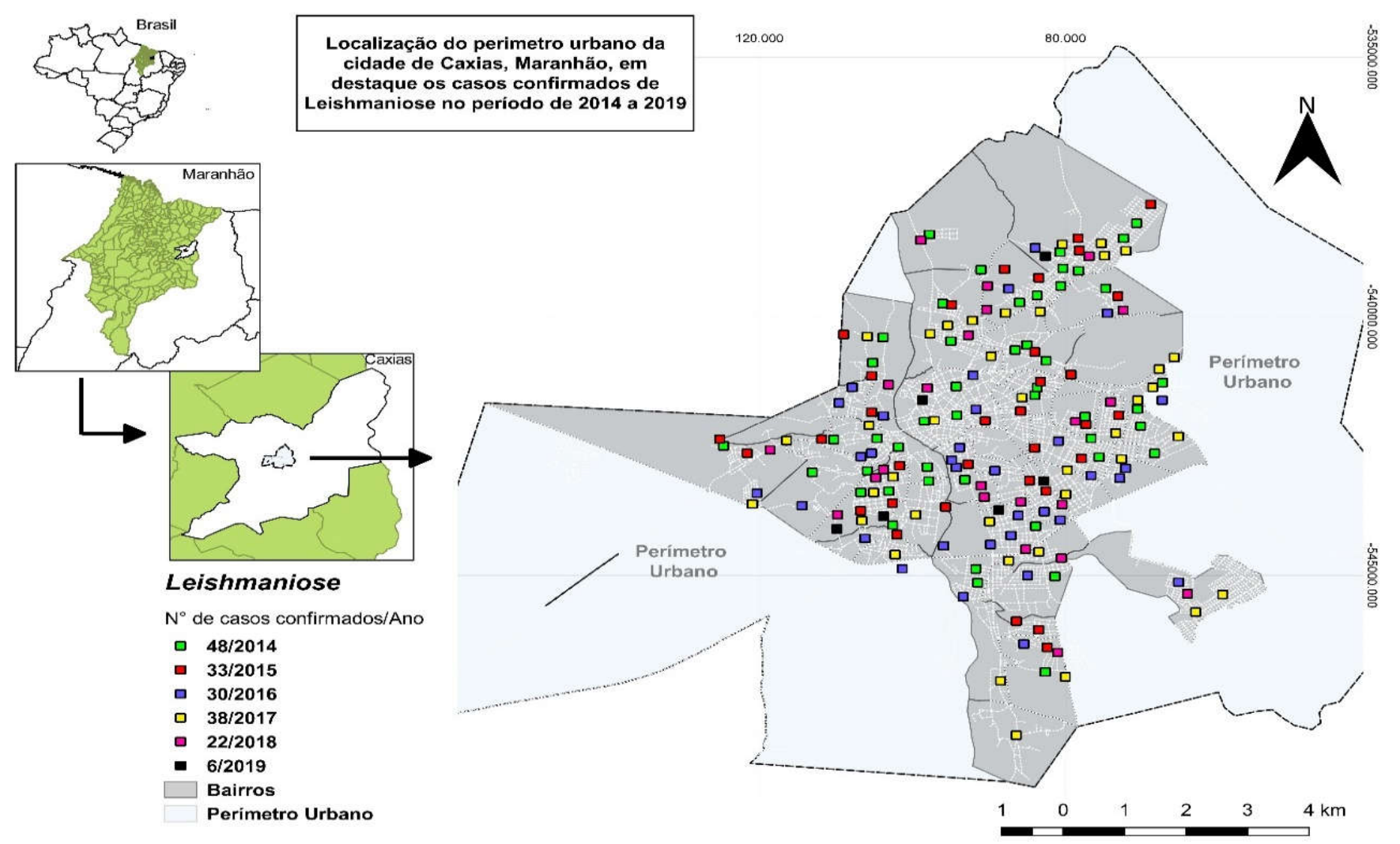

Figura 3. Análise espacial das áreas com casos confirmados de Leishmaniose Visceral Humana em Caxias-MA, $2014-2019$. 
Tabela 2. Características epidemiológicas dos casos notificados de Leishmaniose Visceral Humana em Caxias-MA, 2014-2019

\begin{tabular}{|c|c|c|}
\hline Variáveis & $\mathrm{n}=177$ & $\%$ \\
\hline \multicolumn{3}{|l|}{ Droga inicial utilizada } \\
\hline Antimonial Pentavalente & 147 & 83,1 \\
\hline Anfotericina B & 5 & 2,8 \\
\hline Pentamidina & - & - \\
\hline Anfotericina B lipossoma & 9 & 5,1 \\
\hline Outras & 2 & 1,1 \\
\hline Não utilizada & 8 & 4,5 \\
\hline Em Branco & 6 & 3,4 \\
\hline \multicolumn{3}{|l|}{ Coinfecção com HIV } \\
\hline Sim & 16 & 9,0 \\
\hline Não & 81 & 45,8 \\
\hline Ignorado & 80 & 45,2 \\
\hline \multicolumn{3}{|l|}{ Diagnóstico imunológico } \\
\hline Sim & 62 & 35,0 \\
\hline Não & 23 & 13,0 \\
\hline Ignorado & 92 & 52,0 \\
\hline \multicolumn{3}{|l|}{ Diagnóstico parasitológico } \\
\hline Sim & 41 & 23,2 \\
\hline Não & 14 & 7,9 \\
\hline Ignorado & 122 & 68,9 \\
\hline \multicolumn{3}{|l|}{ Tipo de entrada } \\
\hline Caso novo & 167 & 94,4 \\
\hline Recidiva & 8 & 4,5 \\
\hline Transferência & 2 & 1,1 \\
\hline Ignorado & - & - \\
\hline \multicolumn{3}{|l|}{ Critério de confirmação } \\
\hline Laboratorial & 168 & 94,9 \\
\hline Clínico-epidemiológico & 8 & 4,5 \\
\hline Ignorado & 1 & 0,6 \\
\hline \multicolumn{3}{|l|}{ Evolução do caso } \\
\hline Cura & 105 & 59,3 \\
\hline Abandono & - & - \\
\hline Óbito por LV & 18 & 10,2 \\
\hline Óbito por outras causas & 4 & 2,3 \\
\hline Transferência & 2 & 1,1 \\
\hline Ignorado & 48 & 27,1 \\
\hline
\end{tabular}

evitar a proliferação do mosquito vetor demostrando resultados satisfatórios (Martinset al., 2018), ou devido ao rastreamento dos casos pelos profissionais de saúde ter se tornado mais eficaz (Drumond e Costa, 2011). Acredita-se, até o presente, que os númerosde registros da doença possam ser mais elevados ao considerar a subnotificação de casos (de Sousaet al., 2018). Entretanto a diminuição da ocorrência da LVH paralela à redução da soroprevalência canina parecem apoiar a hipótese de que a identificação e eutanásia dos cães doentes podem contribuir para reduzir as taxas de adoecimento do homem (Wernecket al.,2011). A análise da distribuição da LV quanto ao gênero demostrou que os homens foram os mais acometidos, representando 58,2\%, podendo está atribuída a questões ocupacionais e pela maior exposição aos vetores flebotomíneose não por maior suscetibilidade(Cardimet al., 2013,Fontouraet al., 2016,Souzaet al., 2018). Uma pesquisa realizada em Roraima, aponta que 57,9\% dos casos eram do sexo masculino, corroborando com os resultados desse estudo(Evangelistaet al., 2012). A maioria das pessoas acometidos pela LV tinham baixa escolaridade com até 8 anos de estudo, uma vez que a maioria dos indivíduos foram caracterizados analfabetos ou com ensino fundamental incompleto. Evidenciando-se que a alta prevalência da infecção nesse grupo pode estar associada a menor educação em saúde, o que pode dificultar a captação das orientações sobre prevenção, cuidados necessários e adesão ao tratamento (da Silva Araújo et al., 2019, de Sousa et al., 2018, dos Santos, 2018). Referente a variável faixa etária, a idade compreendida de 1 a 9 anos foi o principal grupo afetado. Estudo conduzido na Ilha de São Luís, Maranhão, encontrou resultado semelhante a este, demostrando que $83,6 \%$ dos casos aconteceram em menores de 9 anos, perfil epidemiológico indicador da proximidade precoce do homem com o cão, principal reservatório urbano e com o vetor da doença (Sousa et al., 2008). No Brasil, a predominância da LV na população infantil permaneceu no decorrer dos anos demonstrando que a doença predominou nos primeiros 5 anos de vida, principalmente em menores de 1 ano, sendo provavelmente devido a susceptibilidade à infecção e imunossupressão deste grupo. A patologia pode estar associada a outros processos infecciosos, inclusive ao vírus daImunodeficiência Adquirida Humana (HIV). Houve significativo aumento do número de pessoas que possuem ao mesmo tempo infecção pelo vírus HIV e pela L.chagasiconfigurando um problema mundial de saúde (Fontouraet al., 2016,de Souza et al.,2020; Souza,2020). Observou-se que as manifestações clínicas encontradas, apresentavam quadro clínicosemelhante ao encontrado em outras doenças infecciosas. Essainespecificidade dos sintomas da LV, dificulta o diagnóstico e tratamento em tempo oportuno, aliado ao potencial lapso de memória e ao baixo nível escolar das populações nas áreas endêmicas, tornando especificamente difícil a interpretação desta informação(Queiroz, 2014).Os sintomas mais frequentes dos casos confirmados foram febres, fraqueza, emagrecimento, palidez e esplenomegalia, sinais clássicos da doença, presentes em quase todos os pacientes no momento de sua internação hospitalar ou intervenção ambulatorial (Cavalcante eVale, 2014,Queiroz,2014). Antes de terem a confirmação diagnóstica da LV, 20\% dos pacientes foram internados, segundo os familiares, devido à pneumonia bacteriana, dengue, virose, esquistossomose, desnutrição e malária(Silvaet al., 2008). A procura por atendimento otimiza o tratamento como também possibilita o diagnóstico precoce (Queiroz et al., 2004,Cavalcante,2014). O munícipio registrou elevada taxa de letalidade, situação vivenciada também no munícipio de Governador Valadares em Minas Gerais que atingiu a taxa de letalidade de $18 \%$ realçando a gravidade da doença (Alves e Fonseca,2018). A expansão da LV vem ocorrendo no espaço e no tempo, com o registro de casos novos em áreas não endêmicas, aumento do número de casos notificados e óbitos, apontando para a necessidade de ações efetivas no controle dessa endemia, sendo que a informação correta, é um instrumento importante para as ações de prevenção e controle da doença (Souza et al., 2008, Cardim et al., 2013). O estado do Maranhão principalmente a região do Leste maranhense, registra o maior número de casos da LV.

Diversos fatores podem ter contribuído para essa disseminação, com destaque para o elevado fluxo migratório, intenso desmatamento, ocupação e urbanização indevida dos espaços, falta de saneamento, e condições ruins de habitação, proporcionando condições favoráveis para manutenção do ciclo de transmissão da doença, realidade presente emmuitos municípios do estado (Furtadoet al., 2015, Fontoura et al., 2016,Garcês Junior et al., 2016,Brasil, 2014). Quanto ao critério de diagnóstico, um número superior a $90 \%$, tiveram confirmação diagnóstica laboratorial de LV, sendo94,4\% considerado como notificações de casos novos,confirmados por meio de exames imunológicos e parasitológicos. Os métodos mais utilizados no país para o diagnóstico de LVH são os sorológicos, principalmente aReação de Imunofluorescência Indireta (RIFI) e o Ensaio Imunoenzimático(ELISA). O teste RIFI apresenta $96 \%$ e $98 \%$ de sensibilidade e especificidade respectivamente, já o ELISA tem sensibilidade de $96 \%$ e $100 \%$ de especificidade (Matoset al. 2015, Brasil, 2019). Neste estudo, 83,1\% dos pacientes com LV, utilizaram o antimonial pentavalente, fármaco utilizado como primeira escolha para tratamento, com a vantagem de poder ser administrado em nível ambulatorial diminuindo assim eventuais riscos relacionados à internação hospitalar.O tratamento dos pacientes com a N-metil glucamina (glucantime), segue protocolos do Ministério da Saúde (MS), abrangendo ainda, medidas adicionais como por exemplo hidratação, antitérmicos, antibióticos, hemoterapia e suporte nutricional.À duração do tratamento deve ser baseada considerando diversos fatores como a evolução clínica, devendo ser observada a velocidade de resposta do paciente como também a presença de comorbidades (Laguardia et al., 2004,Brasil, 2014). A análise espaçotemporal dos mapas demonstroua distribuição geográfica da LV no munícipio de Caxias-MAindicando a prevalência dos casos confirmados da doença de maneira uniformeemtodo o território, mostrando os principais focos, classificando-o como endêmico e de transmissão esporádica. Considerou-se, comoáreas de trabalhoprioritárias local para a realização das ações de vigilância e controle, osbairrosquetiverampelomenos 03 (três) casosoumédia $\geq 1$ nosúltimostrêsanos (Santos, et al., 2019). 


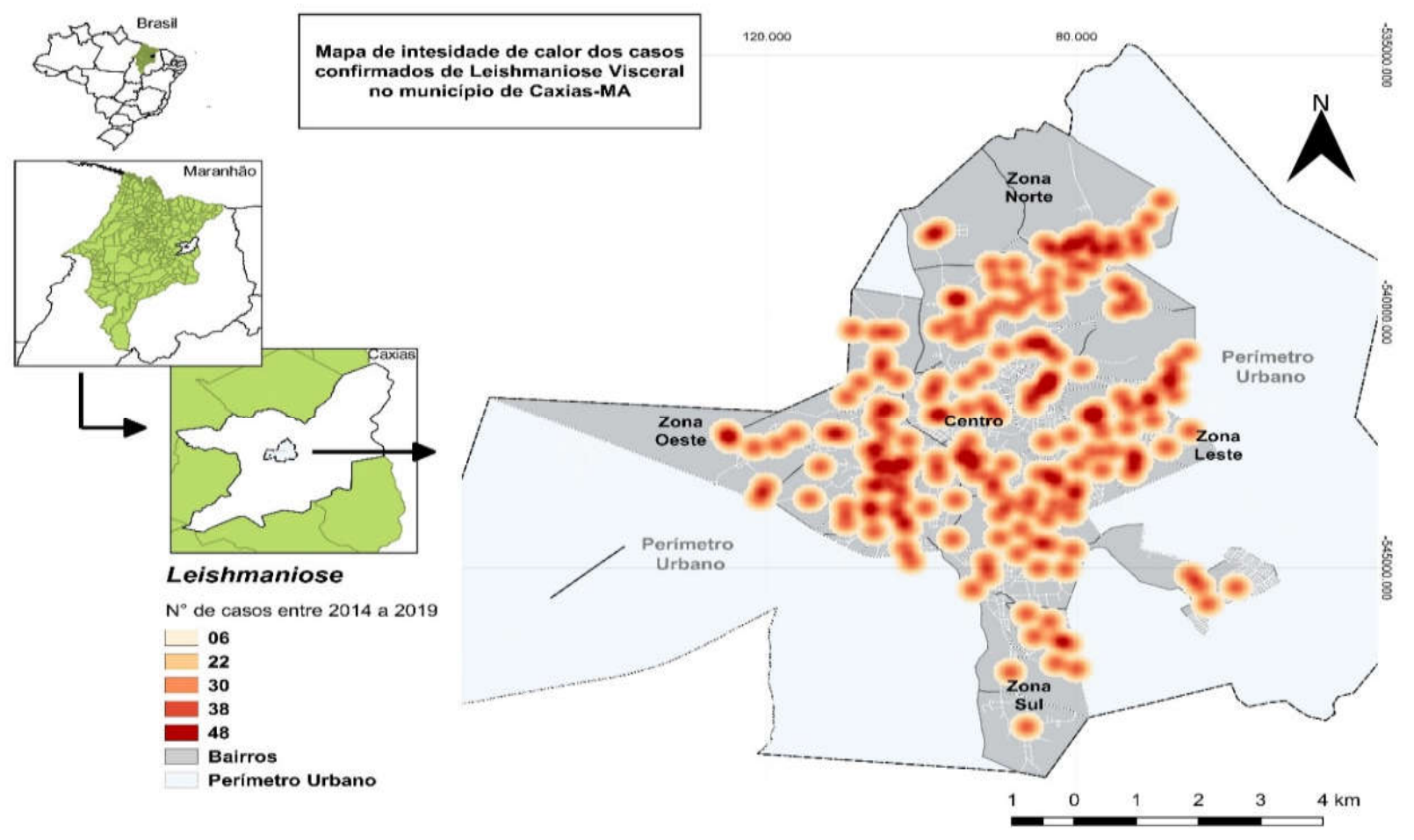

Figura 4. Mapa da intensidade de calor dos casos confirmados de Leishmaniose Visceral Humana em Caxias-MA, 2014-2019

Kawa e Sabroza (2002) demonstraram que o movimento urbano gerou as condições necessárias para a disseminação da doença em focos muito bem definidos, que possibilitaram um contato próximo entre indivíduos suscetíveis e mosquitos vetores. A estratificação de risco é uma importante ferramenta para a vigilância, proporcionando um melhor conhecimento sobre o problema de saúde e adoção de medidas, assim como no direcionamento e priorização de áreas a serem trabalhadas. Entretanto, não há um instrumento acessível e viável para que o serviço de saúde avalie a efetividade das estratégias de controle sobre a transmissão (Brasil,2014; Gontijo e Melo, 2004). Furtado et al. (2015) reportaram a análise espaço-temporal da leishmaniose visceral no estado do Maranhão, registrando que entre 2000 a 2009 foram notificados 5.389 casos de LV, com maiores taxas de incidências nas Unidades Regionais de Saúde (URS): Caxias (36,1/100.000 hab.), Imperatriz (30,8/100.000 hab.), Presidente Dutra (10,8/100.000 hab.), Codó (10,4/100.000 hab.) e Barra do Corda $(9,8 / 100.000$ hab.). No período do referido estudo, o elevado risco relativo nas URS de Imperatriz e Caxias, demonstram mudança no ciclo zoonótico da LV antes considerada tipicamente rural, atingindo claramente áreas urbanas e periurbanas de cidades de médio e grande porte (Brasil,2014, Dantas-torres e Brandão-Filho, 2006). Estudo de Alcântara et al. (2012), evidenciaram a presença de flebotomíneos em galinheiros e pocilgas, associando a prevalência desta doença as condições de higiene e manejo inadequado o que proporciona um ambiente favorável à proliferação do vetor. Estudo de (da Silva et al., 2015) comprovaram a zona urbana de Caxias com abundância da espécie de flebotomíneo de espécimes de L. longipalpis (90\%), principal espécie transmissora da LV. As análises dos dados das notificações, por meio de técnicas de geoprocessamento têm contribuído como ferramenta importante para monitoramento, planejamento e avaliação das ações direcionadas para áreas prioritárias das doenças infecciosas tropicais e reafirmam a urbanização da LV (Margonari, 2006, Garcês-Junior, 2016).

\section{CONCLUSÃO}

Através das análises do perfil clínico-epidemiológico dos casos de LV ocorridos no município de Caxias-MA, pode-se inferir que estas, assemelham-se aos descrito em outros estudos nacionais, uma vez que os dados indicam uma ocorrência endêmica da doença no munícipio por registar casos humanos e caninos. Evidenciando a taxa de $33,1 \%$ $(n=177)$ de casos confirmados de LV com incidência em todo território urbano, acometendo especialmente homens, residentes na zona urbana com baixa escolaridade, e predomínio da infecção na faixa etária compreendida entre 1 a 9 anos. Entende-se que a vulnerabilidade socioeconômica do estado do Maranhão ajuda a responder pela expansão da doença, somando-se a isto a sua grande heterogeneidade espacial. Tendo em vista o padrão de distribuição da $\mathrm{LV}$, a pesquisa revela que o número de casos da doença apresenta-se de maneira uniforme em todo território municipal. A zona urbana, registra elevada taxa da principal espécie transmissora da $L V$, o $L$. longipalpis, sendo encontrados no intra e peridomicilio. $\mathrm{O}$ conhecimento do perfil epidemiológico e análises espaciais poderão nortear a problemática da doença, podendo subsidiar a gestão pública para a atuação e melhor definição de áreas acometidas para direcionamento das ações dirigidas principalmente ao reservatório e vetor. Considera-se importante a implementação de um sistema de monitoramento dessas atividades, porém mantê-las sustentáveis ainda é um desafio devido aos custos decorrentes das ações de vigilância, prevenção e controle. Verificou-se que à incidência de casos de LV em um perfil da população, acometendo grupos específicos e mais suscetíveis, ainda é uma realidade. Apesar das diretrizes bem definidas pelo PVCLV, nota-se que grande parte dos munícipios apresentam dificuldades operacionais, ocasionando baixa efetividade, situação análoga no munícipio de Caxias principalmente referente ao controle, diagnóstico e tratamento em tempo oportuno. Além disso, existem falha no preenchimento das fichas de notificação dos casos o que dificulta caracterizar a real situação. Por fim, é importante compreender que neste estudo existem limitações, dentre elas, o fato de se utilizar dados secundários, erros no diagnóstico e subnotificação de casos, dificultando a implementação das ações da vigilância epidemiológica. Existe a necessidade periódica em se avaliar as ações de prevenção, controle e diagnóstico, com o objetivo de aprimorar a coleta de dados e informações fundamentais para investigação e redução do número de casos e mortalidade ocasionados pela LV.

\section{REFERÊNCIAS}

Alvar, J., Vélez, I. D., Bern, C., Herrero, M., Desjeux, P., Cano, J., ...\& WHO Leishmaniasis Control Team. 2012. Leishmaniasis worldwide and global estimates of its incidence. PloS one, 7(5), e35671.

Alves, W. A., Fonseca, D. S. 2018. Leishmaniose visceral humana: estudo do perfil clínico-epidemiológico na região leste de Minas Gerais, Brasil. Journal of Health \& Biological Sciences, 6(2), 133-139. 
Brasil 2014. Ministério da Saúde. Manual de vigilância e controle da leishmaniose visceral. Ministério da Saúde.

Brasil 2019. Ministério da Saúde. Guia de Vigilância em Saúde/ Ministério da Saúde.

Cardim, M. F. M., Rodas, L. A. C., Dibo, M. R., Guirado, M. M., Oliveira, A. M., \& Chiaravalloti Neto, F. 2013. Introdução e expansão da Leishmaniose visceral americana em humanos no estado de São Paulo, 1999-2011. Revista de Saúde Pública, 47, 691-700.

Cavalcante, Í. J. M., \& Vale, M. R. 2014. Aspectos epidemiológicos da leishmaniose visceral (calazar) no Ceará no período de 2007 a 2011. Revista Brasileira de Epidemiologia, 17, 911-924.

da Silva, L. B., de Aquino, D. M. C., Leonardo, F. S., Guimarães, A. S., Melo, M. N., Rebêlo, J. M. M., \& Pinheiro, V. C. S. 2015. Flebotomíneos (Diptera, Psychodidae) em focos urbanos de leishmaniose visceral no Estado do Maranhão, Brasil. Revista de Patologia Tropical/Journal of Tropical Pathology, 44(2), 181194.

de Sousa, N. A., Linhares, C. B., Pires, F. G. B., Teixeira, T. C., da Silva Lima, J., \& Nascimento, M. D. L. O. 2018. Perfil epidemiológico dos casos de leishmaniose visceral em Sobral-CE de 2011 a 2015. SANARE-Revista de Políticas Públicas, 17(1).

de Souza, H. P., de Oliveira, W. T. G. H., dos Santos, J. P. C., Toledo, J. P., Ferreira, I. P. S., de Sousa Esashika, S. N. G., \& de Sousa Delácio, A. 2020. Doenças infecciosas e parasitárias no Brasil de 2010 a 2017: aspectos para vigilância em saúde. Revista Panamericana de Salud Publica, 44, e10.

dos Santos, G. M. 2018. Características epidemiológicas da leishmaniose tegumentara mericana em um estado do nordestebrasileiro. ARCHIVES OF HEALTH INVESTIGATION, $7(3)$.

Da Conceição Araújo, D. 2017. Análise espacial dos casos humanos de leishmaniose visceral. Arquivos de Ciências da Saúde, 24(2), 71-75.

da Silva Araújo, D. B., de Souza Nascimento, E., Coelho, V. A. T., de Souza, C. G., \& Pereira, M. C. S. 2019. Perfil sociodemográfico da leishmaniose tegumentar americana em Almenara-Minas Gerais. PUBVET, 14, 139.

de Alcântara, S. H., de Assunção Sousa, M. F., Magalhães, N. A., \&Terto, G. G. 2012. Características epidemiológicas e distribuição espacial da Leishmaniose Visceral Americana canina e humana na Cidade de Caxias, no Estado do Maranhão de 2005 a 2010\&. PUBVET, 6, Art-1472.

Dantas-Torres, F., \& Brandão-Filho, S. P. 2006. Expansão geográfica da leishmaniose visceral no Estado de Pernambuco. Revista da Sociedade Brasileira de Medicina Tropical, 39(4), 352-356.

Drumond, K. O., \& Costa, F. A. L. 2011. Forty years of visceral leishmaniasis in the State of Piaui: a review. Revista do Instituto de Medicina Tropical de São Paulo, 53(1), 3-11.

Evangelista, L. S. D. M., \&Sibajev, A. (2012). Perfil epidemiológico da leishmaniose visceral no estado de Roraima. BEPA. Boletim Epidemiológico Paulista (Online), 9(102), 30-35.

Fontoura, I. G., Fontoura, V. M., \&Nascimento, L. F. C. 2016. Análise espacial da ocorrência de leishmaniose visceral no estado do Tocantins, Brasil. Ambiente \&Água-An Interdisciplinary Journal of Applied Science, 11, 1088-1095.

Furtado, A. S., Nunes, F. B. B. D. F., Santos, A. M. D., \& Caldas, A. D. J. M. (2015). Space-time analysis of visceral leishmaniasis in the State of Maranhão, Brazil. Ciencia \& saude coletiva, 20, 3935-3942.

Garcês Junior, A. R., Brasil, G. V. D. S., Ferreira, L. C., Rodrigues, Z. M. R., \& Aquino Júnior, J. 2016. Análise dos casos de leishmaniose visceral utilizando técnicas de geoprocessamento. $J$. health inform, 929-938.

Gontijo, C. M. F., \&Melo, M. N. 2004. Leishmaniose visceral no Brasil: Quadro atual, desafios e perspectivas. RevistaBrasileira de Epidemiologia, 7(3), 338-349.

Kawa, H., \& Sabroza, P. C. 2002. Espacialização da leishmaniose tegumentar na cidade do Rio de Janeiro. Cadernos de Saúde Pública, 18(3), 853-865.
Laguardia, J., Domingues, C. M. A., Carvalho, C., Lauerman, C. R., Macário, E., \&Glatt, R. 2004. Sistema de informação de agravos de notificação em saúde (Sinan): desafios no desenvolvimento de um sistema de informação em saúde. Epidemiologia e Serviços de Saúde, 13(3), 135-146.

Lana, R. S., Michalsky, É. M., Lopes, L. O., Lara-Silva, F. O., Nascimento, J. L., Pinheiro, L. C., \& Dias, E. S. 2018. Ecoepidemiological aspects of visceral leishmaniasis in an endemic area in the Steel Valley in Brazil: An ecological approach with spatial analysis. PloS one, 13(10), e0206452.

Marcondes, M., \& Rossi, C. N. 2013. Leishmaniose visceral no Brasil. Brazilian Journal of Veterinary Research and Animal Science, 50(5), 341-352.

Margonari, C., Freitas, C. R., Ribeiro, R. C., Moura, A. C. M., Timbó, M., Gripp, A. H., \& Dias, E. S. 2006. Epidemiology of visceral leishmaniasis through spatial analysis, in Belo Horizonte municipality, state of Minas Gerais, Brazil. Memórias do Instituto Oswaldo Cruz, 101(1), 31-38.

Martins, C. P., Brandão, M. G. S. A., de Mesquita Braga, M., Sampaio, L. B. F., Barros, L. M., \& Pacheco, J. C. B. (2018). Monitoramento epidemiológico como instrumento de apoio à gestão de saúde: análise das notificações de leishmaniose visceral emSobral, Ceará. Revista de AdministraçãoemSaúde, 18(72).

Matos, H. J. D., Pinto, A. Y. D. N., Miranda, A. M. M., Silva, F. L. C., \& Ramos, F. L. D. P. 2015. Reação cruzada nos testes sorológicos entre doença de Chagas e leishmaniose visceral emregiões endêmicas para ambas as doenças. Revista PanAmazônica de Saúde, 6(1), 65-68.

OPAS-Organização Pan-americana da Saúde 2019. Leishmanioses. Informe epidemiológico das américas. Relatório de leishmaniose $\mathrm{n}^{\mathrm{o}} 7.2019$.

Queiroz, M. J., Alves, J. G., \&Correia, J. B. (2004). Leishmaniose visceral: características clínico-epidemiológicas em crianças de área endêmica. Jornal de Pediatria, 80(2), 141-146.

Silva, A. R. D., Tauil, P. L., Cavalcante, M. N. S., Medeiros, M. N., Pires, B. N., \&Gonçalves, E. D. G. D. R. (2008). Situação epidemiológica da leishmaniose visceral, nallha de São Luís, Estado do Maranhão. Revista da Sociedade Brasileira de Medicina Tropical, 41(4), 358-364.

Souza, C. L. N. D., Luz, Z. P., \&Rabello, A. 2008. Análise da informação sobre a leishmaniose visceral disponível em portais brasileiros da rede mundial de computadores: internet. Revista da Sociedade Brasileira de Medicina Tropical, 41(4), 352-357.

SANTOS, A. N. dos et al. 2019. Vigilância epidemiológica/ Unidade de Vigilância de Zoonoses atenção primária e vigilância em saúde. Plano de ação para a intensificação da vigilância e controle da Leishmaniose Visceral no município de Caxias-Ma para o ano de 2020.

Souza, E. C., Braga, K. L., da Silva, T. K., \& de Lira Silva, M. 2020. Apresentação clínica da leishmaniose visceral em pacientes portadores do HIV: Análise dos Fatores Relacionados ao Aparecimento da Doença. Brazilian Journal of Health Review, 3(2), 1766-1777.

Viana, G. M. D. C., Silva, M. A. C. N. D., Garcia, J. V. D. S., Guimarães, H. D., Arcos Júnior, G. F., Santos, A. V. A., ... \&Galvão, C. D. S. 2017. Epidemiological profile of patients coinfected with visceral leishmaniasis and HIV/AIDS in Northeast, Brazil. Revista da Sociedade Brasileira de Medicina Tropical, 50(5), 613-620.

Zuben, A. P. B. V., \&Donalísio, M. R. 2016. Dificuldades na execução das diretrizes do Programa de Vigilância e Controle da Leishmaniose Visceral em grandes municípios brasileiros. Cadernos de Saúde Pública, 32, e00087415.

Werneck, G. L., Hasselmann, M. H., \& Gouvêa, T. G. (2011). Panorama dos estudos sobre nutrição e doenças negligenciadas no Brasil. Ciência \& Saúde Coletiva, 16, 39-62.

WHO 2020. Leishmaniose Situação Epidemiológica. Epidemiologia. 2018.

WHO 2020. LeishmanioseVisceral. 